\title{
An Ultra-Wideband Low-Noise Amplifier for 3-5-GHz Wireless Systems
}

\author{
Ahmad Saghafi \\ Dept. of Electrical Engineering \\ Malek Ashtar University of Technology \\ Tehran 15875-1774, Iran \\ Email: saghafi.a@gmail.com
}

\author{
Abdolreza Nabavi \\ Dept. of Electrical Engineering \\ Tarbiat Modares University \\ Tehran 14115-143, Iran \\ Email: abdoln@modares.ac.ir
}

\begin{abstract}
In this paper, an ultra-wideband low-noise amplifier is designed and simulated in a 0.13- $\mu \mathrm{m}$ CMOS technology for a 3 5-GHz UWB system. For ultra-wideband operation, shunt-series feedback topology is used. To improve noise performance, the amplifier employs inductive load. Biasing point variation which occurs due to the resistive feedback is fixed by adding a capacitor in series with feedback. Thus, the desirable gain is achieved with a lower power consumption. Simulations show a $-3-d B$ gain bandwidth of $6 \mathrm{GHz}$ between $2 \mathrm{GHz}$ and $8 \mathrm{GHz}$, a minimum noise figure of $1.9 \mathrm{~dB}$ in the 3-5-GHz band, a power gain of 11.5 dB while consuming $13.9 \mathrm{~mW}$.
\end{abstract}

\section{INTRODUCTION}

Ultra-wideband (UWB) technology uses narrow pulses to transmit information over a wide frequency band. As approved by the Federal Communication Commission (FCC) in 2002 [1], a UWB system must occupy at least $500 \mathrm{MHz}$ of bandwidth between $3.1 \mathrm{GHz}$ and $10.6 \mathrm{GHz}$ and transmit maximum equivalent isotropic radiated power (EIRP) spectral density of $-41.3 \mathrm{dBm} / \mathrm{MHz}$. Owing to the low power of UWB signals it can coexist with other systems that use the same frequency band. This technology has many applications such as wireless personal area networks (WPANs) which is currently developing by the IEEE standard group, sensor networks, and localizers [2].

Unique advantages of UWB systems such as high data rate, low-power consumption, immunity to multipath environments, and coexistence with narrow band systems are due to the very narrow pulses and low power of the transmitted signals. However, these characteristics, in turn, introduce new challenges in the design of UWB receivers. Since a UWB system employs very narrow pulse, its equivalent spectrum will spread over a wide frequency band with extremely low power. One of the most critical component in the analog front-end, the LNA, requires to amplify the received signal with sufficient gain and as little additional noise as possible. Design of a broadband LNA with matched input impedance, flat gain, and good noise performance over the entire frequency spectrum is a very difficult task.

In this paper, a low-noise amplifier for use in ultra-wideband systems is designed and analyzed. The LNA employs shuntseries feedback topology to enjoy its broad band behavior and good input and output characteristics [3]. To further increase the gain and bandwidth, cascode common source architecture is employed. A capacitor is used in series with feedback to avoid the effect of the output voltage on the optimum biasing point. Therefore, the desirable gain is achieved with a low power consumption. To improve the output noise performance, an inductive load which also overcomes the gain degradation at higher frequencies is employed. Another inductor is added in series with feedback to give additional gain at higher frequencies.

Simulation of the LNA in a $0.13-\mu \mathrm{m}$ CMOS technology illustrates a power gain of $11.5 \mathrm{~dB}$ in the $-3-\mathrm{dB}$ gain bandwidth of $2-8 \mathrm{GHz}$, a minimum noise figure of $1.9 \mathrm{~dB}$ in the $3-5-\mathrm{GHz}$ band, and a power consumption less than $14 \mathrm{~mW}$.

The reminder of the paper is organized as follows. Section II discusses the LNA design. Section III describes more accurate considerations in the design of ultra-wideband LNAs. Section IV presents the results of the simulations, and Section V concludes the paper.

\section{LNA DESIGN}

The inductive degeneration topology is often used for the design of narrow band LNAs [3], [4]. The most important advantage of this topology is low noise figure due to the existence of inductor in the drain and source of the transistor. However, the inductor in the source of the transistor causes the LNA to show narrow band characteristics. Hence, this topology cannot satisfy the wideband requirements needed for ultra-wideband LNAs.

Shunt-series feedback topology can match the input impedance in a broad bandwidth. Also, it presents a good noise performance. However, due to the presence of the resistive feedback, the biasing point of the LNA is fixed with the output voltage. Therefore, the biasing cannot be set to the optimal point. Consequently, to achieve the desired gain, the system consumes more power than the case of having optimal biasing point. Moreover, the architecture has the stability problem due to feedback. Motivated by some good characteristics of this topology such as low-noise performance and wideband behavior, we have chosen this architecture and in the subsequent sections, after analyzing a simplified circuit, we propose a new architecture based on the shunt-series feedback topology which overcomes the above problems. 


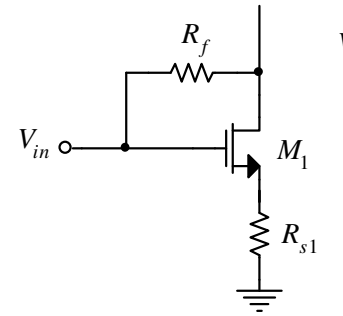

(a)

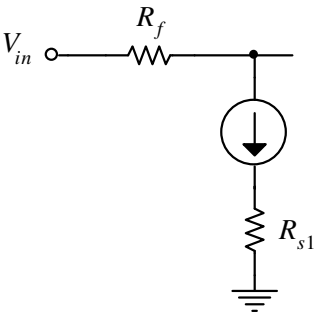

(b)
Fig. 1. Shunt-series feedback amplifier. (a) LNA circuit, (b) small signal equivalent circuit.

\section{A. Shunt-Series Feedback LNA Analysis}

A simplified architecture of a shunt-series feedback LNA is shown in Fig 1(a). H-parameters can be calculated from the small-signal model shown in Fig. 1(b) as

$$
[H]=\left[\begin{array}{cc}
R_{f} & 1 \\
\frac{g_{m} R_{f}}{1+g_{m} R_{s 1}} & \frac{g_{m}}{1+g_{m} R_{s 1}}
\end{array}\right]
$$

Using the standard equations, H-parameters can be converted to S-parameters as follows

$$
[S]=\frac{1}{\Delta}\left[\begin{array}{cc}
\frac{R_{f}}{Z_{0}}-\frac{g_{m} Z_{0}}{1+g_{m} R_{s 1}} & 2 \\
2\left(1-\frac{g_{m} R_{f}}{1+g_{m} R_{s 1}}\right) & \frac{R_{f}}{Z_{0}}-\frac{g_{m} Z_{0}}{1+g_{m} R_{s 1}}
\end{array}\right]
$$

Where $\Delta=S_{11} S_{22}-S_{12} S_{21}$ and $Z_{0}$ is the characteristic impedance of the system. In ideal matching condition, i.e., $S_{11}=S_{22}=0, R_{s 1}$ can be calculated as

$$
R_{s 1}=\frac{Z_{0}^{2}}{R_{f}}-\frac{1}{g_{m}}
$$

Substituting (3) onto (2) gives S-parameters with the assumption of ideal matching:

$$
[S]=\left[\begin{array}{cc}
0 & \frac{Z_{0}}{R_{f}+Z_{0}} \\
1-\frac{R_{f}}{Z_{0}} & 0
\end{array}\right]
$$

As shown in (4), by choosing appropriate value for $R_{s 1}$ and $R_{f}$, flat gain, $S_{21}$ can be achieved. Since the value of $R_{s 1}$ cannot be negative, (3) limits $g_{m}$ by the following equation

$$
g_{m} \geq \frac{R_{f}}{Z_{0}^{2}}=\frac{1-S_{21}}{Z_{0}}
$$

Thus, the desirable gain determines the minimum value of $g_{m}$. If (5) does not satisfy, the system will oscillate. Due to parasitic capacitance and inductance that cannot be neglected at higher frequencies, the gain is degraded. Also, because the biasing point is not adjusted to the optimum voltage, the LNA requires higher power consumption to achieve the desired gain. Therefore, this simple architecture of shunt-series feedback topology needs some modifications to overcome the mentioned problems.

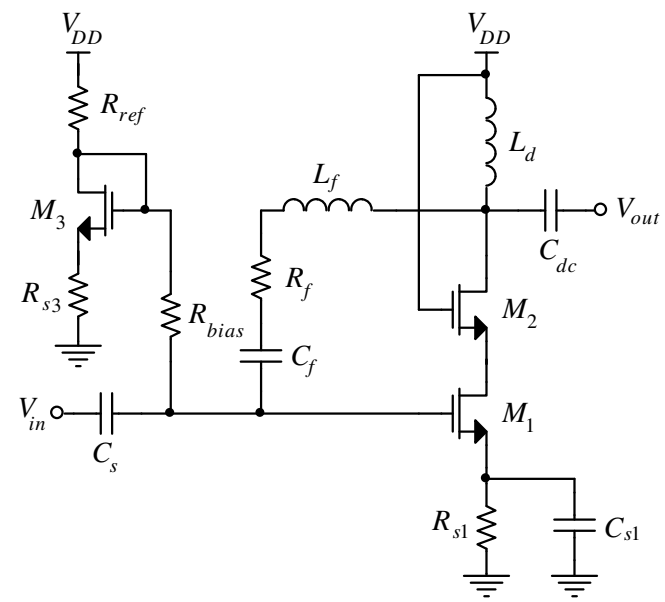

Fig. 2. Modified shunt-series feedback ultra-wideband low-noise amplifier with cascode topology.

\section{B. The Proposed LNA Architecture}

Fig. 2 shows the modified architecture of the shunt-series feedback LNA. Cascode topology is used instead of simple common source to increase the gain and the bandwidth, and to improve the stability of the system [3], [4]. Also, the frequency compensated matching network technique, described in Section III-B, is applied to improve the gain flatness of the system in the desired frequency band [5].

In Fig. 2, the inductor, $L_{d}$, replaces the resistive load. This inductor decreases the output noise and also compensates for the gain degradation of the LNA at higher frequencies. This compensation occurs, because the magnitude of the inductor's impedance increases as frequency increases. Regarding to the manufacturing limitations, $L_{d}$ cannot be large enough to achieve the wideband response. Therefore, to achieve a higher gain and bandwidth, an inductor, $L_{f}$, is added. Although, by increasing $R_{f}$ the LNA gain increases, (5) limits the value of $R_{f}$, whereas increasing the inductor, $L_{f}$, has not any effect on (5).

As mentioned before, feedback causes the biasing point to vary by the output voltage. Due to this nonoptimal biasing point, the LNA has to consume a higher power to achieve the desirable gain. Because of this, the capacitor, $C_{f}$, is added to separate the output and the input voltage levels. Therefore, the optimal biasing point can be acheived. To bias the circuit, transistor, $M_{3}$, is employed. This transistor and transistor $M_{1}$ form a current mirror source and its channel width is some fractions of $M_{1}$ 's channel width to minimize the power overhead of the bias circuit [3].

\section{More AcCurate Considerations IN THE UWB LNA DESIGN}

The analysis presented in the previous section is based on low-frequency models. However, at higher frequencies, the effects of parasitic capacitors and inductors should be taken into consideration. For a more accurate design, some important 
issues such as stability, constant gain, and noise figure should be exactly investigated.

\section{A. LNA Stability}

Stability factor of a system can be defined with S-parameters as [4]

$$
k=\frac{1+|\Delta|^{2}-\left|S_{11}\right|^{2}-\left|S_{22}\right|^{2}}{2\left|S_{12}\right|\left|S_{21}\right|}
$$

To have an unconditional stable system, the following equations must be simultaneously satisfied

$$
k>1, \Delta<1
$$

To put the system in the unconditionally stable region, one can add a series resistor or a shunt conductance to the input or output port [6]. Adding a series resistor will increase the output noise, due to the thermal noise of the resistor in the signal path. Therefore, it is better to add a shunt conductance to the port. This conductance should be small enough to produce a little equal current noise while stabilizes the LNA.

\section{B. Constant Gain}

Transduced gain is usually used in RF design as defined below [3]

$$
G_{T}=\frac{\text { Power delivered to the load }}{\text { Available power from the source }}
$$

The above definition can be described with S-parameters and the the reflection coefficients of the source and the load of the LNA as

$$
G_{T}=\frac{\left|S_{21}\right|^{2}\left(1-\left|\Gamma_{S}\right|^{2}\right)\left(1-\left|\Gamma_{L}\right|^{2}\right)}{\left|\left(1-S_{11} \Gamma_{S}\right)\left(1-S_{22} \Gamma_{L}\right)-S_{12} S_{21} \Gamma_{L} \Gamma_{S}\right|^{2}}
$$

where $\Gamma_{S}$ and $\Gamma_{L}$ are the source and the load reflection coefficients, respectively. In UWB LNA design, having a flat gain is more important than maximum gain achievement which is exactly unlike the narrow band design that is required to achieve maximum gain. To maximize the gain, $\Gamma_{S}$ and $\Gamma_{L}$ should be zero. However, in UWB systems, reflection coefficients are designed to fix $G_{T}$ in a broad frequency band. In other word, at some frequencies $G_{T}$ is reduced to keep the gain flatness. This method is called frequency-compensated matching or selective mismatching [5].

Frequency-compensated matching method is a simple way to decrease the gain by not perfectly matching the transistor to its load. In other word, there is no perfectly impedance matching in the bandwidth of the system. To use this method, constant gain circles should be plotted on a Smith chart. For a UWB LNA, the constant gain circles must be plotted on a single chart at some frequencies over the bandwidth of the system. By changing the feedback elements, such as $R_{f}, L_{f}$, and $C_{f}$, constant gain circles can be moved to overlap at somewhere near the centre of the Smith chart. In this case, the gain of the UWB LNA is flat over the operational frequency range. Fig. 3 shows constant gain circles of the proposed LNA over the $3-5-\mathrm{GHz}$ band. The circles are plotted at the frequencies of 3,4, and $5 \mathrm{GHz}$. As can be seen, the circles overlaps near the centre of the chart and the resulting gain in Fig. 4 shows only $0.5 \mathrm{~dB}$ variation in the $3-5-\mathrm{GHz}$ band.

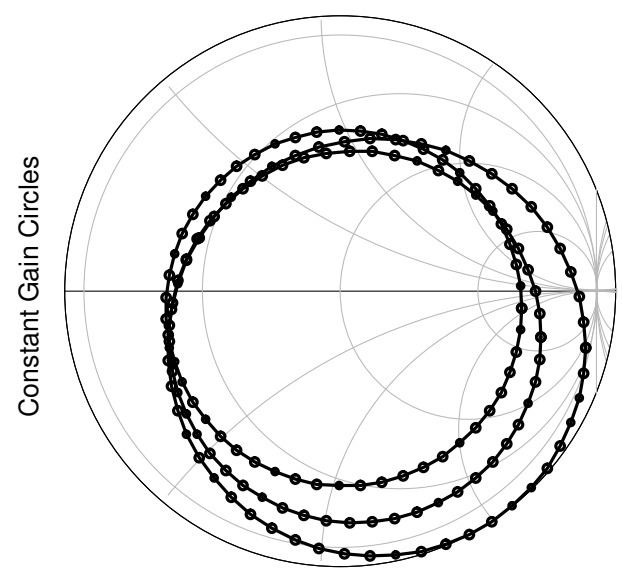

Fig. 3. Constant gain circles at $11.5 \mathrm{~dB}$ over the $3-5-\mathrm{GHz}$ band.

\section{Noise Considerations}

One of the most important noise sources is the input resistor of the power supply. Since, in a UWB system there is no perfectly impedance matching, reflected signals generate noise. The other important noise sources are thermal noises which are generated by the feedback resistor and transistor. The two major noise types in transistor are Flicker noise and thermal noise. Since, a UWB system has a wide frequency range, Flicker noise is neglected. There are two thermal noise sources in the transistor. First, the drain noise generated in the channel and second, the thermal noise generated by the gate resistor which can be decreased with proper layout technique.

As a result, the most important noise sources in the LNA can be summarized as: power supply noise, thermal noise of the feedback resistor and transistor noise which is generated in the channel. A higher feedback resistors decreases its thermal noise. Also, increasing the channel width decreases the channel noise.

\section{Simulation Results}

The proposed UWB LNA is simulated using Agilent ADS in a $0.13-\mu \mathrm{m}$ CMOS technology. The supply voltage is $V_{D D}$ $=1.6 \mathrm{~V}$. The channel width of the transistors are $W_{1}=W_{2}=$ $10 W_{3}=550 \mu \mathrm{m}$. The biasing current is set to $I_{D 1}=10 I_{D 3}$ $=7 \mathrm{~mA}$. A $3-\mathrm{nH}$ inductor, $L_{d}$, is used as the load of the LNA. Considering the gain flatness, bandwidth and stability, feedback components are chosen as: $R_{f}=350 \Omega, L_{f}=2 \mathrm{nH}$, and $C_{f}=400 \mathrm{fF}$. In a $50-\Omega$ system, a $2-\mathrm{k} \Omega$ resistor is adequate for $R_{\text {bias }}$ which results in a negligible noise current.

Fig. 4 shows the simulated S-parameters of the designed LNA. The maximum power gain of the amplifier is $11.5 \mathrm{~dB}$ in a $-0.5-\mathrm{dB}$ gain bandwidth of $3-5 \mathrm{GHz}$ and the $-3-\mathrm{dB}$ gain bandwidth is $6 \mathrm{GHz}$ between $2 \mathrm{GHz}$ and $8 \mathrm{GHz}$. Noise figure versus frequency variation is shown in Fig. 5 which attains a minimum of $1.9 \mathrm{~dB}$ in the $3-5-\mathrm{GHz}$ band. Fig. 5 also shows the stability factor, $k$, and $\Delta$, versus frequency. As can be seen, (7) is satisfied for the entire bandwidth, Thus, the LNA is unconditionally stable. 
TABLE I

COMPARISON OF THE PROPOSED UWB LNA WITH PUbLISHED WORKS

\begin{tabular}{c|c|c|c|c|c|c|c}
\hline Ref. & $\mathrm{BW}_{3-d B}(\mathrm{GHz})$ & $\mathrm{G}_{\max }(\mathrm{dB})$ & $\mathrm{NF}_{\min }(\mathrm{dB})$ & DC Supply $(\mathrm{V})$ & Power $(\mathrm{mW})$ & Technology & Year \\
\hline \hline$[7]^{*}$ & $1.5 \sim 7.5$ & 7 & 8.7 & 3 & 216 & $0.6-\mu \mathrm{m}$ CMOS & 2002 \\
\hline$[8]^{*}$ & $1 \sim 7$ & 13.1 & 3.3 & 1.8 & 75 & $0.18-\mu \mathrm{m}$ CMOS & 2003 \\
\hline$[9]^{*}$ & $2 \sim 4.6$ & 9.8 & 2.3 & 1.8 & 12.6 & $0.18-\mu \mathrm{m}$ CMOS & 2005 \\
\hline$[10]^{*}$ & $3 \sim 6$ & 15.91 & 4.7 & 1.8 & 59.4 & $0.18-\mu \mathrm{m}$ CMOS & 2005 \\
\hline This work $^{* *}$ & $2 \sim 8$ & 11.5 & 1.9 & 1.6 & 13.9 & $0.13-\mu \mathrm{m}$ CMOS & 2006 \\
\hline
\end{tabular}

${ }^{*}$ Experimental. $\quad{ }^{* *}$ Simulation.

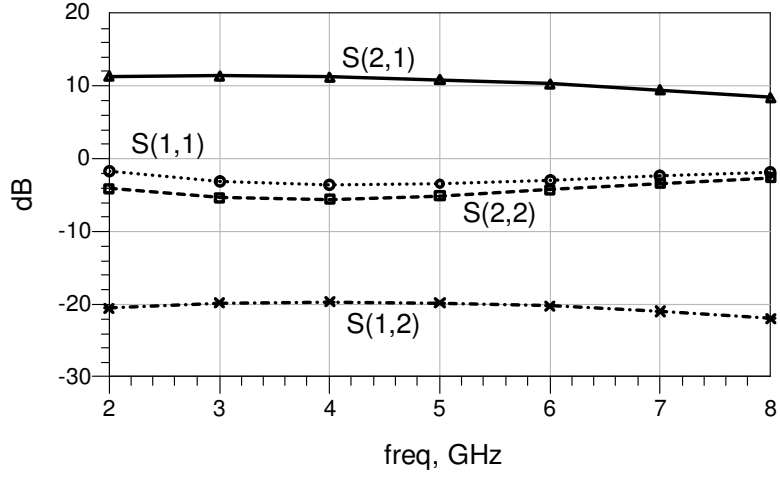

Fig. 4. S-parameters of the proposed LNA.

Table I compares the simulation results of this work with some previously reported works. For a better comparison we have chosen references [7]-[10] since the bandwidth of the LNA in these works are near to the bandwidth of the LNA in our work.

\section{CONCLUSION}

The proposed ultra-wideband LNA architecture is based on the resistive shunt-series feedback topology. To improve noise performance, the amplifier employs inductive load. Simulations using a $0.13-\mu \mathrm{m}$ CMOS technology show a $-3-\mathrm{dB}$ gain bandwidth of $6 \mathrm{GHz}$ between $2 \mathrm{GHz}$ and $8 \mathrm{GHz}$, a minimum noise figure of $1.9 \mathrm{~dB}$ in the $3-5-\mathrm{GHz}$ band, and a power gain of $11.5 \mathrm{~dB}$ with $13.9 \mathrm{~mW}$ power consumption.

\section{REFERENCES}

[1] Federal Communications Commission, "Revision of part 15 of the commissions rules regarding ultra-wideband transmission systems: First report and order," Tech. Rep., FCC, Apr. 2002.

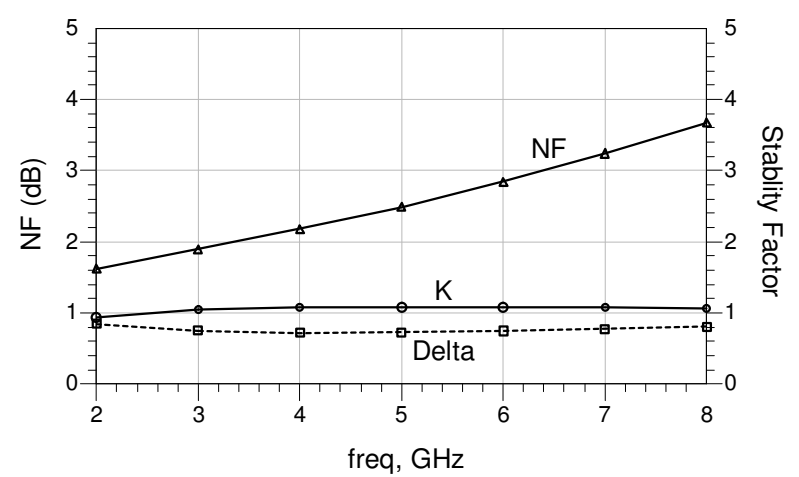

Fig. 5. Noise figure and stability factor of the proposed LNA.

[2] D. Porcino and W. Hirt, "Ultra-wideband radio technology: Potential and challenges ahead," IEEE Commun. Mag., vol. 31, no. 7, pp. 66-73, Jul. 2003.

[3] T. H. Lee, The Design of CMOS Radio-Frequency Integrated Circuits, 2nd ed. New York: Cambridge Univ. Press, 2004.

[4] B. Razavi, RF Microelectronics, Prentice Hall, Upper Saddle River NJ, 1998.

[5] K. W. Kobayashi et al., "GaAs heterojunction bipolar transistor MMIC DC to $10 \mathrm{GHz}$ direct-coupled feedback amplifier,' In Proc. GaAs IC Symposium, 1989, pp. 87-90.

[6] K. Yamamoto et al., "A 2.4-GHz-band 1.8-V operation single-chip SiCMOS T/R-MMIC front-end with a low insertion loss switch," IEEE J. Solid-State Circuits, vol. 36, pp. 1186-1197, Aug. 2001.

[7] H.-T. Ahn and D. J. Allstot, "A $0.5-8.5-\mathrm{GHz}$ fully differential CMOS distributed amplifier," IEEE J. Solid-State Circuits, vol. 37, pp. 985-993, Aug. 2002.

[8] S. Andersson, C. Svensson, and O. Drugge, "Wideband LNA for a multistandard wireless receiver in $0.18 \mu \mathrm{m} \mathrm{CMOS,"} \mathrm{in} \mathrm{Proc.} \mathrm{ESSCIRC,}$ Sep. 2003, pp. 655-658.

[9] C.-W. Kim et al., "An Ultra-Wideband CMOS Low Noise Amplifier for 3-5 GHz UWB System," IEEE J. Solid-State Circuits, vol. 40, pp. 11861197, Feb. 2005.

[10] C.-P. Chsng and H.-R. Chuang, "0.18 $\mu \mathrm{m}$ 3-6 GHz CMOS broadband LNA for UWB radio," IEEE Electronics Letters, vol. 41, no. 12, June 2005 . 